\title{
Operational Amplifier with 1-V Rail-to-Rail Multipath-Driven Output Stage
}

\author{
Jeroen Fonderie and Johan H. Huijsing, Senior Member, IEEE
}

\begin{abstract}
A bipolar operational amplifier (op amp) with a rail-to-rail multipath-driven output stage that operates at supply voltages down to $1 \mathrm{~V}$ is presented. The bandwidth of this output stage is as high as possible, viz. equal to that of one of the output transistors, loaded by the output capacitance. The output voltage can reach both supply rails within $100 \mathrm{mV}$ and the output current is $\pm 15 \mathrm{~mA}$. The op amp is designed to be loaded by a 100-pF capacitor and the unity-gain bandwidth is $3.4 \mathrm{MHz}$ at a $60^{\circ}$ phase margin. The voltage gain is $117 \mathrm{~dB}$ and the CMRR is $100 \mathrm{~dB}$. The frequency behavior of the multipathdriven (MPD) topology has an improved performance when compared to that of previously presented low-voltage output stages. A figure of merit $F_{M}$ for low-voltage op amps has been defined as the bandwidth-power ratio.
\end{abstract}

\section{INTRODUCTION}

$\mathrm{T}$ HE TREND towards very low supply voltages forces new demands on the design of accurate analog build ing blocks. An important building block is the operational amplifier (op amp). Op amps that run on a supply voltage of $1 \mathrm{~V}$ have already been in existence for over a decade [1], but until now, the bandwidth of these circuits has been in the kilohertz range rather than in the megahertz range. The bandwidth that can be obtained is mainly limited by the output stage of the low-voltage op amp. To be universally applicable, the output stage has to meet the following requirements.

1) Usually, base-emitter voltages prevent the output from reaching either one of the supply rails. However, to be able to deliver an output voltage signal that is as large as possible, preferably from rail to rail, the actual output transistors have to be connected in a common-emitter (CE) configuration.

2) The minimum supply voltage should preferably be as low as $1 \mathrm{~V}$. This enhances the applicability of the op amp, such as operation on a one-cell battery, but it hampers the design of the op amp because only one base-emitter voltage fits in the supply-voltage range.

3) The maximum bandwidth of the op amp is determined by the current flowing through the output transistors, which determines their transconductance, and by the

Manuscript received May 5, 1991; revised September 16, 1991. This work was supported by Signetics Company, Sunnyvale, CA, and by the Technology Foundation, The Netherlands.

The authors are with the Department of Electrical Engineering, Delft University of Technology, 2628 CD Delft, The Netherlands.

IEEE Log Number 9104189. capacitive load it has to drive. Any transistors preceding the actual CE output transistors more or less reduce the obtainable bandwidth. This reduction should be kept as small as possible.

4) The output stage should be able to push and pull output currents in the order of $10 \mathrm{~mA}$, which means that its current gain should be in the order of $10^{3}$. One output transistor in $\mathrm{CE}$ configuration does not meet this condition and therefore emitter followers are normally placed in front of it.

5) Finally, the power consumption must be kept low, so the quiescent current, which is drawn from the supplies by the output stage, must be as small as possible. Also, the output stage must be realized with few and small transistors in order to keep the chip size limited.

The op amp presented here has a complementary class$\mathrm{AB}$ output stage that very well meets the demands listed above. It further contains a complementary input stage with a rail-to-rail common-mode input range and an intermediate stage that is insensitive to the Early effect if the supply voltage is much higher than the minimum value of $1 \mathrm{~V}$.

To be able to compare the output stage presented here with the prior art, both are discussed with respect to the requirements mentioned above. In Section II, two previously presented output stages are examined, and Section III deals with the output stage presented here. To verify the theory of Sections II and III, three op amps with three different output stages have been integrated. The circuit implementation is discussed in Section IV. Section $\mathrm{V}$ covers the measurement results and in Section VI conclusions are drawn.

\section{Darlington and Widlar Output Stage}

The circuit shown in Fig. 1 is the well-known Darlington output stage. For simplicity, only the n-p-n or pull side is shown, but the theory presented in this and the following section also applies to the complementary p-n-p side. The driver transistor $Q_{2}$ precedes the output transistor $Q_{1}$ and the intermediate stage consists of transistors $Q_{11}-Q_{14}$. Miller capacitor $C_{m 1}$ splits the pole at the output of the intermediate stage and the one at the output of the output stage. A difficult condition, where the frequency compensation is concerned, is that the 


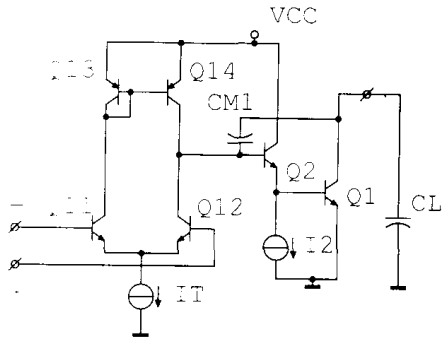

Fig. 1. The n-p-n Darlington output stage.

circuit can be loaded by a relatively large output capacitor $C_{L}$.

With respect to the specifications summed up in the Introduction, the following analysis of the Darlington output stage can be made.

1) Since the output transistor has a CE configuration, the output-voltage range is large; depending on the output current, $Q_{1}$ is able to reach the supply rail within about $100 \mathrm{mV}$.

2) The minimum value of the supply voltage is not $1 \mathrm{~V}$ but $1.8 \mathrm{~V}$, because the two base-emitter voltages of $Q_{1}$ and $Q_{2}$ are stacked.

3) As in all CE output stages, the bandwidth of the Darlington output stage is determined by the quiescent current through $Q_{1}$ and by $C_{L}$, but, unfortunately, it is limited by the internal pole of the output stage, located at the base of $Q_{1}$. If the output current increases, the output bump in the frequency response is likely to occur [2]. This bump becomes even more likely in the design of op amps employing lateral p-n-p transistors, where the bandwidth is limited by the transit frequency of the transistors, as well as by the load capacitor. Unfortunately, the use of $p$-n-p's in the CE configuration is inevitable in low-voltage op amps.

4) The current gain of the Darlington output stage is equal to $\beta_{1} \beta_{2}$, if $\beta$ is the current gain of the transistors $Q_{1}$ and $Q_{2}$, respectively. Even if the output stage consists of lateral p-n-p transistors with a moderate current gain of 30 , the total current gain of the output stage is large enough to push output currents in the order of milliamperes.

5) The Darlington output stage consists of only two transistors and the transistor $Q_{2}$ consumes only $1 / \beta$ of the current through $Q_{1}$, which makes this output stage very efficient. However, to avoid the output bump, the quiescent current through $Q_{2}$ should be substantially increased.

Another previously presented output stage is the Widlar output stage, shown in Fig. 2 [1]. Widlar used the output stage in National Semiconductor's 1-V op amp LM10. Output transistor $Q_{1}$ is preceded by the emitter followers $Q_{2}$ and $Q_{3}$. To be able to pull large output currents, a boost circuit $Q_{4}-Q_{6}$ is added. The intermediate stage comprises transistors $Q_{11}-Q_{14}$ and the circuit is Miller compensated by $C_{m 1}$.

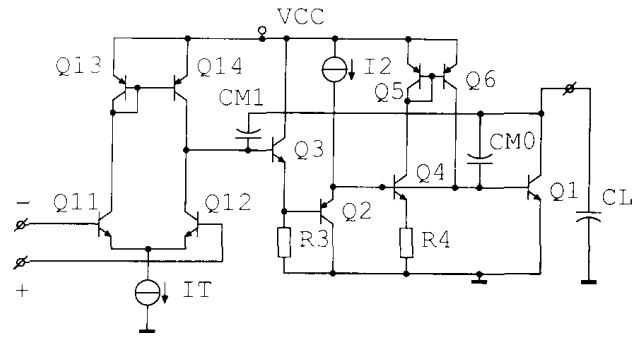

Fig. 2. The n-p-n Widlar output stage.

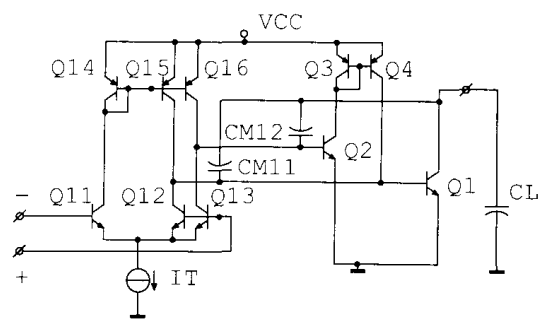

Fig. 3. The n-p-n MPD output stage.

If the Widlar output stage is also investigated regarding the previously stated requirements, the following results are found.

1) Transistor $Q_{1}$ has a $\mathrm{CE}$ configuration, so the output-voltage range is again from rail to rail.

2) The emitter followers $Q_{2}$ and $Q_{3}$ are folded, yielding a minimum supply voltage of only $1 \mathrm{~V}$.

3) The input signal has to pass through emitter followers $Q_{2}$ and $Q_{3}$, which lowers the obtainable bandwidth and makes the occurrence of the output bump even more likely. We found that the positive loop gain around $Q_{4}-Q_{6}$ and the presence of $Q_{2}$ and $Q_{3}$ necessitates the insertion of an additional compensation capacitor $C_{m 0}$ [3], to keep the output bump within bounds at high current levels; this further reduces the bandwidth.

4) The current gain of the Widlar output stage is potentially very high. If the boost circuit $Q_{4}-Q_{6}$ is configured appropriately, a current gain of more than $10^{4}$ is possible.

5) When designed for optimal bandwidth, the current through $Q_{2}-Q_{4}$ can easily be half that flowing through $Q_{1}$, giving a rather high total current consumption. The output stage consists of seven transistors, of which $Q_{2}$ and $Q_{3}$ must have a rather large emitter area, and an additional capacitor. Because of this, the chip size is larger than that of the Darlington output stage.

\section{Multipath-Driven Output Stage}

Fig. 3 shows the n-p-n multipath-driven (MPD) output stage [4]. Output transistor $Q_{1}$ is driven by $Q_{2}-Q_{4}$. Because this signal path provides the necessary gain, it is referred to as the "gain path." Parallel to the gain path, the so-called "feedforward path" directly drives output 
transistor $Q_{1}$. This feedforward path gives the circuit the maximal obtainable bandwidth and bypasses the bandwidth reduction caused by $Q_{2}-Q_{4}$. The clue to this success is that the intermediate stage, with transistors $Q_{11}-Q_{16}$, supplies the output stage with two identical, but decoupled signals. The pole at the output of $Q_{1}$ and the two poles at the two outputs of the intermediate stage are being split by the Miller capacitors $C_{m 11}$ and $C_{m 12}$, respectively.

With respect to the demands on low-voltage op amps as summarized in the Introduction, the following is claimed about the op amp with MPD output stage.

1) The MPD output stage has a rail-to-rail output-voltage range.

2) Since none of the base-emitter voltages of the transistors are stacked, the output stage operates at a supply voltage of $1 \mathrm{~V}$.

3) The frequency behavior of the MPD output stage equals that of the capacitively loaded output transistor $Q_{1}$. The gain path through $Q_{2}-Q_{4}$ does not influence the frequency behavior because of the feedforward path to $Q_{1}$. The bandwidth is now entirely determined by the current through $Q_{1}$ and by $C_{L}$.

4) The current gain of the MPD output stage is of the same order as that of the Darlington output stage.

5) The MPD output stage consists of four transistors, but also two additional transistors are needed in the intermediate stage; it therefore occupies more chip area than the Darlington but less than the Widlar output stage. The output stage is economical; driver transistors $Q_{2}-Q_{4}$ only consume $2 / \beta$ of the current flowing through $Q_{1}$.

To confirm these claims, the behavior of the MPD output stage is further analyzed. The first claim speaks for itself, and the second can be verified directly from Fig. 3. To investigate the third claim about the frequency behavior of the MPD output stage, the transfer from the input voltage of the intermediate stage to the output voltage is analyzed. To calculate this transfer, the current equations of the small-signal equivalent circuit are solved. The resulting voltage gain of the circuit is

$$
\frac{U_{o}}{U_{i}}=\frac{1}{s r_{e 13} C_{m 12}} \cdot \frac{1+s \frac{r_{e 13}}{r_{e 12}} r_{e 2} C_{m 12}}{1+s r_{e 2} C_{m 11}+s^{2} r_{e 2} C_{m 11} r_{e 1} C_{L}}
$$

where $r_{e}$ are the emitter resistances of the transistors and $s$ is the complex frequency, and the Miller capacitors $C_{m 11}$ and $C_{m 12}$ are assumed large compared to the diffusion capacities of the transistors. Since

$$
r_{e 2} \approx \beta_{1} r_{e 1}
$$

it can be assumed that

$$
r_{e 2} C_{m 11}=r_{e 1} \beta_{1} C_{m 11} \gg r_{e 1} C_{L} .
$$

If, further, the Miller capacitors $C_{m 11}$ and $C_{m 12}$ as well as the current through the intermediate transistors $Q_{12}$ and $Q_{13}$ are equal:

$$
r_{e 13} C_{m 12}=r_{e 12} C_{m 11}
$$

and the voltage gain becomes

$$
\frac{U_{o}}{U_{i}}=\frac{1}{s r_{e 13} C_{m 12}} \cdot \frac{1}{1+s r_{e 1} C_{L}} .
$$

The pole-zero cancellation depends on the matching of the Miller capacitors and of intermediate stage transistors. This can be done very accurately, which is important, because a pole-zero doublet gives a slow settling time of the transient response [5]. Fig. 4 shows the frequency response of the intermediate and output stage. The dashed and dotted lines represent the response of the gain and feedforward paths, respectively; the gain path has a large gain, but a smaller bandwidth, and the feedforward path has a small gain, but larger bandwidth. As can be seen from Fig. 4, no pole-zero doublet occurs. The bandwidth is, thus, only limited by the current flowing through $Q_{1}$ and by the load capacitor $C_{L}$.

The current gain of the MPD output stage can be calculated as $\frac{1}{2} \beta_{1}\left(\beta_{2}+1\right) \approx \frac{1}{2} \beta_{1} \beta_{2}$. If necessary, it could be further increased by scaling the current mirror $Q_{3}, Q_{4}$. Note that $Q_{4}$ supplies just the current needed to drive output transistor $Q_{1}$; in particular, this implies that, at small output currents, only a very small current flows through $Q_{2}-Q_{4}$.

The fifth assertion, regarding the number of transistors and their current consumption, can again be verified directly from Fig. 3.

\section{Circuit Implementation}

The MPD output stage shown in Fig. 5 embodies two complementary parts, $Q_{110}-Q_{140}$ and $Q_{210}-Q_{240}$. Both parts are identical to the output stage shown in Fig. 3, except that the current mirrors $Q_{130}-Q_{140}$ and $Q_{230}-Q_{240}$ are scaled $1: 4$ to increase the gain. The small emitter resistors $R_{110}$ and $R_{210}$ prevent breakthrough of the output transistors at high current and voltage levels, but they do not limit the output-voltage range in normal operation; when driving light loads, the output voltage is able to reach within $100 \mathrm{mV}$ of the supply rails. The output transistors $Q_{110}$ and $Q_{210}$ are able to sink or source a maximum output current of $15 \mathrm{~mA}$. Transistors $Q_{150}$ and $Q_{250}$ are part of the class-AB current control loop. The quiescent current through the output transistors is kept at $320 \mu \mathrm{A}$, and the minimum current through either one of these transistors is $160 \mu \mathrm{A}$.

Fig. 6 shows the input stage, the intermediate stage, and the class- $\mathrm{AB}$ current control. The intermediate stage consists of two parts, each with its own tail current source of $22 \mu \mathrm{A}$, and it has four outputs to drive the four inputs of the output stage. The differential stage $Q_{410}-Q_{420}$ supplies the n-p-n output stage and has n-p-n current mirrors $Q_{450}-Q_{460}$ that are connected to the negative supply rail, just as the n-p-n output transistors are. The other differential stage $Q_{430}-Q_{440}$ is connected to the p-n-p output stage and has p-n-p current mirrors $Q_{470}-Q_{480}$ that are connected to the positive supply rail. Thus, the voltages on all matching transistors of the 

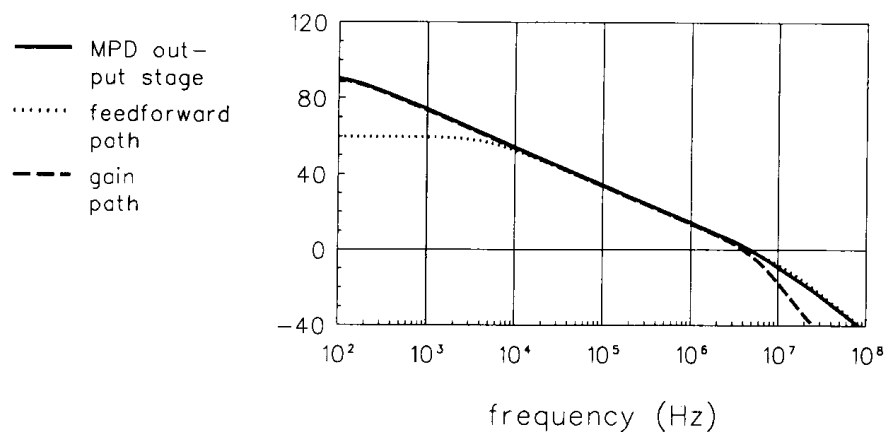

Fig. 4. Frequency response of the MPD output stage. The dashed line gives the response of the gain path, with a large gain but smaller bandwidth, and the dotted line marks the response of the feedforward path, which has a small gain but larger bandwidth.

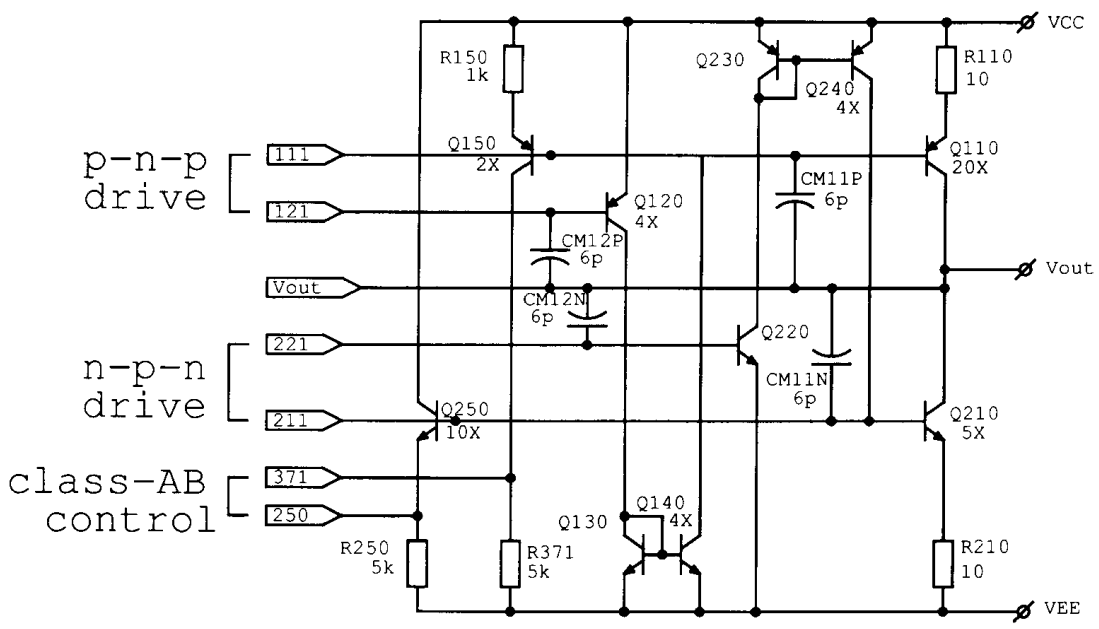

Fig. 5. Circuit implementation of the complementary MPD output stage.

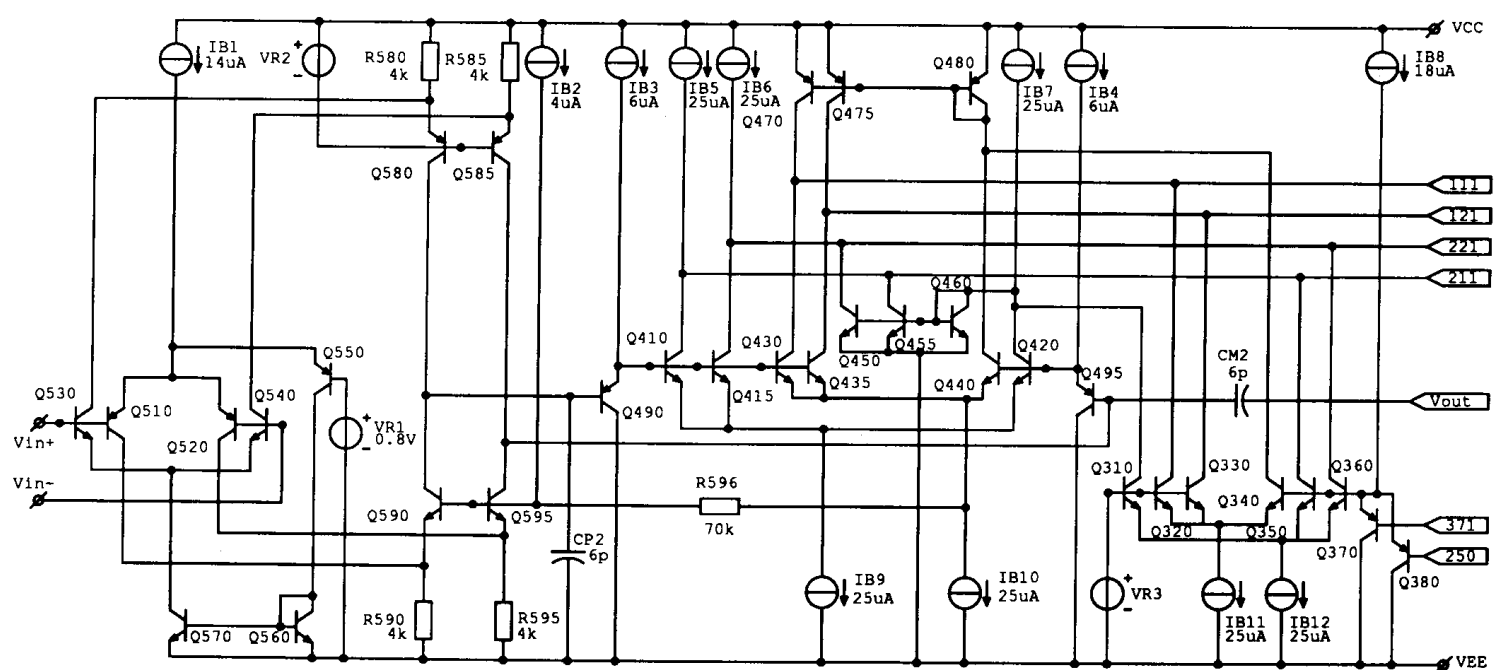

Fig. 6. Circuit implementation of the input stage, intermediate stage, and class-AB current control. 
intermediate stage are equal and the Early effect is compensated. This guarantees operation also when the supply voltage is much higher than the $1 \mathrm{~V}$ minimum.

The feedback class-AB current control embodies transistors $Q_{150}$ and $Q_{250}$, shown in Fig. 5, and $Q_{310}$ through $Q_{380}$, shown in Fig. 6. The differential amplifier $Q_{310}-Q_{360}$ consists of two parts, each connected to the matching part of the intermediate stage, again to compensate for the Early effect. The currents through the output transistors $Q_{110}$ and $Q_{210}$ are converted into voltages at the bases of $Q_{370}$ and $Q_{380}$ by $Q_{150}$ and $Q_{250}$, respectively. These voltages are compared to the reference voltage $V_{R 3}$ by the feedback amplifier $Q_{310}-Q_{360}$ and thus the quiescent current through $Q_{110}$ and $Q_{210}$ is kept at a constant value. If one of the output transistors is supplying an output current, the matching transistor of the pair $Q_{370}, Q_{380}$ is cut off, and the current through the other, nonactive, output transistor is regulated at half the quiescent current by the feedback amplifier $Q_{310}-Q_{360}[6]$, [7].

At supply voltages of $1.7 \mathrm{~V}$ and more, the input stage $Q_{510}-Q_{540}$ has a rail-to-rail input-voltage range. For supply voltages below that level, only the p-n-p input pair operates in the common-mode range from the negative supply rail to $0.8 \mathrm{~V}$ below the positive supply. However, if desired, an input stage with a fully rail-to-rail commonmode input range at a supply voltage of $1 \mathrm{~V}$ could also be incorporated [3]. Current switch $Q_{550}$ keeps constant the sum of the currents through both input pairs over the full common-mode input range. The transconductance of the input stage therefore has a constant value of $0.25 \mathrm{mmho}$. The input-offset voltage changes between that of the $n-p-n$ and p-n-p transistor pair when the common-mode input voltage crosses the turnover range of $Q_{550}$. This limits the CMRR, as is described in Section V. The circuit $Q_{580}-Q_{595}$ adds the currents of the differential input stages.

The current sources and reference voltages included in the op amp are realized with the bias circuit shown in Fig. 7. It comprises a PTAT current source $Q_{10}-Q_{60}$ and a start-up circuit $Q_{70}-Q_{80}[8]$.

The output is assumed to be loaded by a maximum output capacitor $C_{L}$ of $100 \mathrm{pF}$. At a quiescent current through the output transistors of $320 \mu \mathrm{A}$, the output transconductance $g_{\text {out }}$ is $12 \mathrm{mmho}$, and the base-emitter capacitance $C_{b e}$ is 0.6 and $2 \mathrm{pF}$ for the n-p-n and $\mathrm{p}-\mathrm{n}-\mathrm{p}$ transistors, respectively. The Miller capacitors $C_{m 11 P}$, $C_{m 12 P}, C_{m 11 N}$, and $C_{m 12 N}$, with a value of $6 \mathrm{pF}$ each, are connected between the output of the output stage and each of its four inputs. The output pole then has a value:

$$
\frac{g_{\text {out }}}{2 \pi C_{L}\left(1+C_{b e 1} / C_{m 11}\right)} \approx 15 \mathrm{MHz} \text {. }
$$

The bandwidth of the output and intermediate stage should be chosen at a factor of 2 lower than the output pole [7], which is realized with an intermediate-stage transconductance $g_{\text {int }}$ of 0.28 mmho:

$$
\frac{g_{\mathrm{int}}}{2 \pi C_{m 11}}=\frac{1}{2} \frac{g_{\mathrm{out}}}{2 \pi C_{L}\left(1+C_{b e 1} / C_{m 11}\right)} \approx 7.5 \mathrm{MHz}
$$

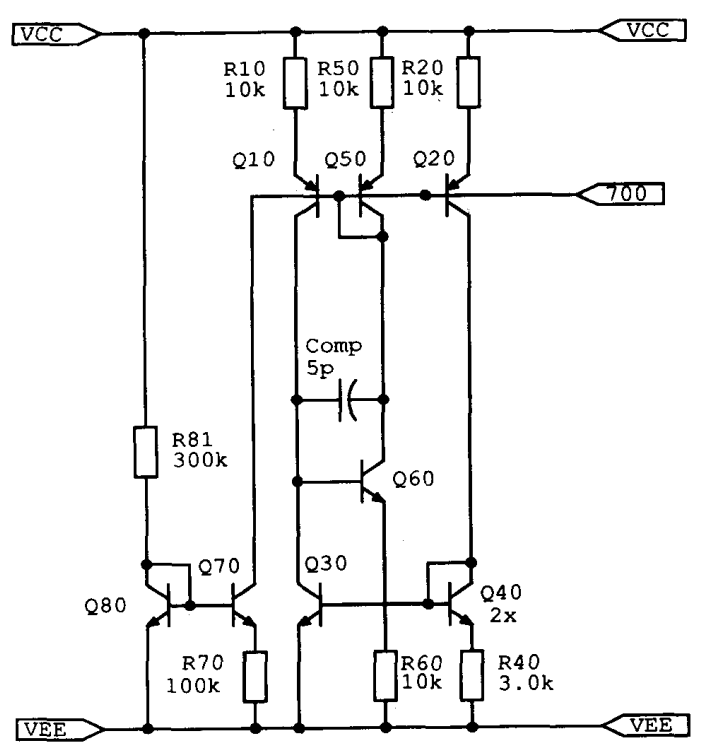

Fig. 7. The PTAT current source.

The overall bandwidth should again be half this value, which is achieved with a Miller capacitor $C_{m 2}$ of $6 \mathrm{pF}$, connected between the output of the output stage and the output of the input stage, and an input-stage transconductance $g_{\text {inp }}$ of $0.25 \mathrm{mmho}$ :

$$
\begin{aligned}
\frac{g_{\text {inp }}}{4 \pi C_{m 2}} & =\frac{1}{2} \frac{g_{\text {int }}}{2 \pi C_{m 11}}=\frac{1}{4} \frac{g_{\text {out }}}{2 \pi C_{L}\left(1+C_{b e 1} / C_{m i 1}\right)} \\
& =3.4 \mathrm{MHz} .
\end{aligned}
$$

The op amp now has a straight 6-dB/octave frequency rolloff from $3 \mathrm{~Hz}$ to $6 \mathrm{~dB}$ below the unity-gain frequency of $3.4 \mathrm{MHz}$. Any pole-zero doublet, which would remain in the MPD output stage, is effectively suppressed by this second nest.

\section{Measurements}

Besides the MPD output stage, a Darlington and Widlar output stage have also been integrated with the same input and intermediate stages shown in Fig. 5. Fig. 8 shows a micrograph of the op amps with MPD and Darlington output stages. On the right-hand side of the micrograph the output stages can be distinguished; in the middle, the intermediate stage and class- $A B$ current control are situated. The input stage, with quad layout, can be seen to the left of the micrograph. The total capacity used in each of these op amps is $41 \mathrm{pF}$. The chip area that is occupied by the output stages is $0.26 \mathrm{~mm}^{2}$ for the MPD output stage and $0.20 \mathrm{~mm}^{2}$ for the Darlington output stage. A micrograph of the op amp with the Widlar output stage is shown in Fig. 9. Because of the additional compensation capacitors, the total capacity is $51 \mathrm{pF}$ for the Widlar output stage and the chip area is $0.30 \mathrm{~mm}^{2}$. The op amps have been made in a $12-\mathrm{V}$ 


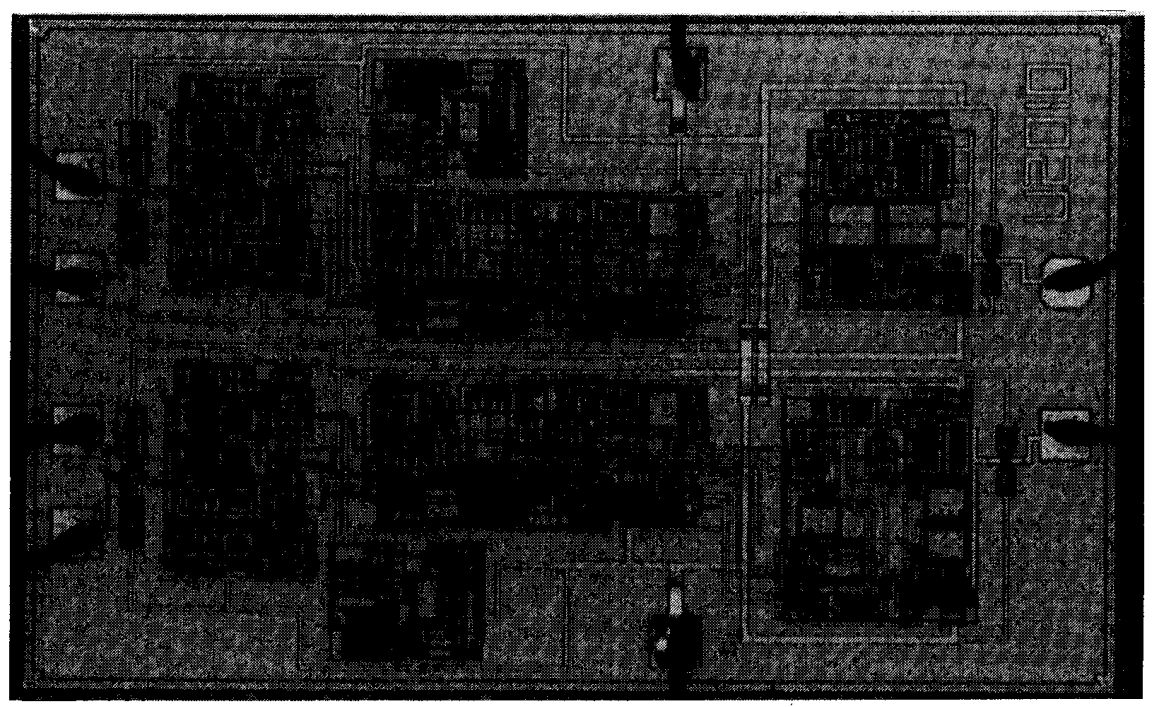

Fig. 8. Micrograph of the op amp with MPD output stage (bottom) and the op amp with Darlington output stage (top).

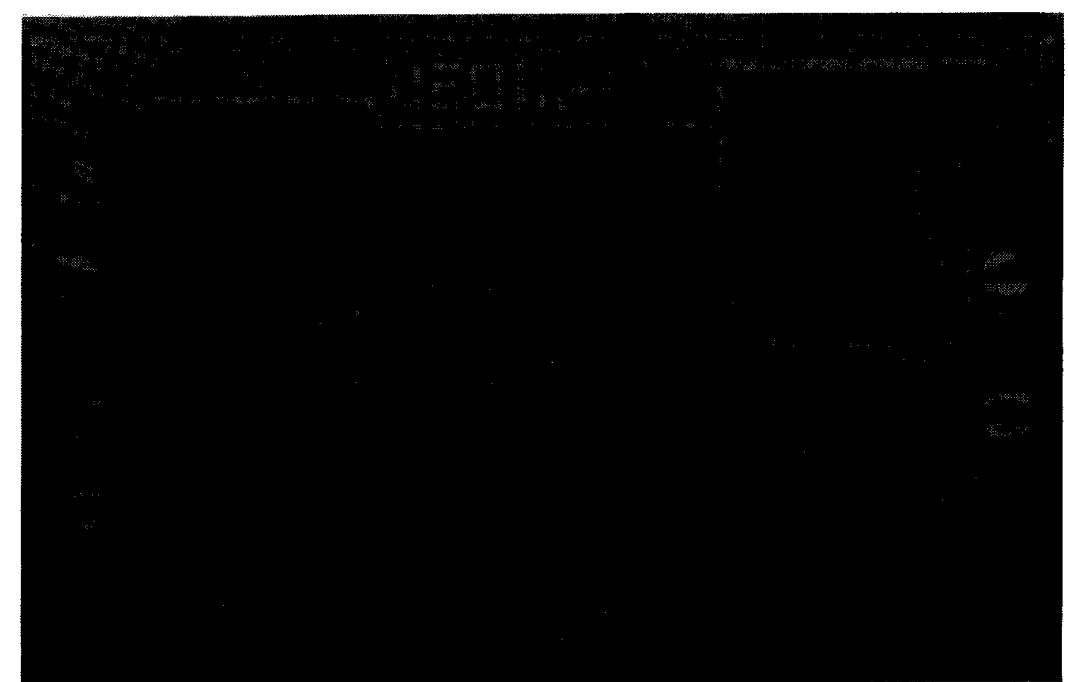

Fig. 9. Micrograph of the op amp with Widlar output stage (bottom). The circuit shown on top of the micrograph is not discussed here.

BiCMOS process with 3-GHz n-p-n transistors and 1-GHz p-n-p transistors.

The measured open-loop frequency response of the op amp with MPD output is shown in Fig. 10. The supply voltage is $1 \mathrm{~V}$ and the op amp is loaded by a $100-\mathrm{pF}$ capacitor and a $10-\mathrm{k} \Omega$ resistor in parallel. The unity-gain frequency is $3.4 \mathrm{MHz}$ at a phase margin of $61^{\circ}$, and the dc gain is $117 \mathrm{~dB}$. If the op amp drives a light load, the phase margin increases to $70^{\circ}$.

Since all op amps have been designed with an equal quiescent current of $320 \mu \mathrm{A}$ flowing through the output transistors, the bandwidth of all three, following from (8), is roughly the same. The supply power needed to achieve this differs, however. To compare the performance of the op amps, a figure of merit $F_{M}$ is therefore defined as the bandwidth-power ratio of the output stages:

$$
F_{M}=\frac{B_{W}}{V_{\text {sup }_{\min }} \cdot I_{\text {sup }}}
$$

The dimension of this figure of merit $F_{M}$ is inverse to the well-known power-delay product $P_{d}$ in digital circuits. Table I shows the values of $F_{M}$ of the three output stages together with the characteristics from which they are derived. The MPD output stage has a substantially higher 


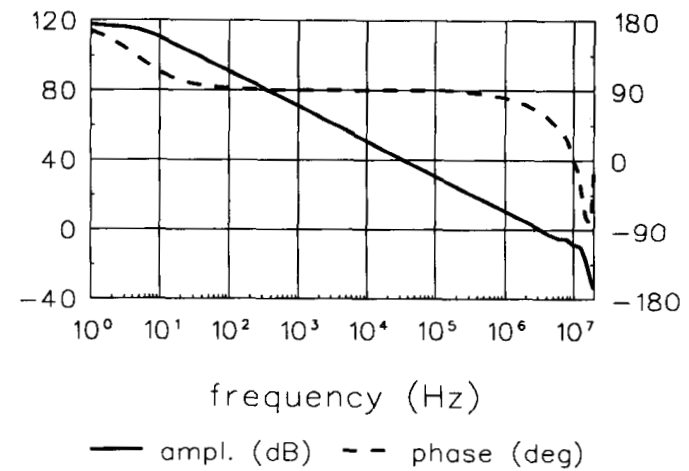

Fig. 10. Measured frequency response of the op amp with MPD output stage. The unity-gain frequency is $3.4 \mathrm{MHz}$, and the unity-gain phase margin is $61^{\circ}$.

TABLE I

Figure of MERIT, $F_{M}=B_{w} / V_{\text {sup }_{\text {min }}} I_{\text {sup }}$, FOR THE Three OUTPUT STAGES

\begin{tabular}{|c|c|c|c|c|}
\hline Parameter & MPD & Darlington & Widlar & Unit \\
\hline Figure of merit $F_{M}$ & 18 & 11 & 9.6 & $\mathrm{MH}_{z} / \mathrm{mW}$ \\
\hline Bandwidth Output Stage & 7.5 & 7.5 & 6.7 & $\mathrm{MHz}$ \\
\hline Minimum Supply Voltage & 1.0 & 1.8 & 1.0 & $\mathrm{~V}$ \\
\hline $\begin{array}{l}\text { Supply Current Output } \\
\text { Stage }\end{array}$ & 420 & 395 & 695 & $\mu \mathrm{A}$ \\
\hline
\end{tabular}

TABLE II

SPeCifications of the Complete Op Amps

\begin{tabular}{|c|c|c|c|c|}
\hline \multicolumn{4}{|c|}{$\begin{array}{l}\text { MPD: Op Amp with Multipath-Driven Output Stage, } 1.0-\mathrm{V} \\
\text { Darlington: Op Amp with Darlington Output Stage, } 2.0-\mathrm{V} \mathrm{S} \\
\text { Widlar: Op Amp with Widlar Output Stage, } 1.0-\mathrm{V} \text { Supply } \\
\text { All Op Amps: } R_{L}=10 \mathrm{k} \Omega, C_{L}=100 \mathrm{pF} \text {, Temp }=27^{\circ} \mathrm{C}\end{array}$} & \multirow[b]{2}{*}{ Unit } \\
\hline Parameter & MPD & Darlington & Widlar & \\
\hline \multicolumn{5}{|l|}{ Input-Offset } \\
\hline Voltage & 0.6 & 0.6 & 0.6 & $m V$ \\
\hline \multicolumn{5}{|l|}{ Input-Noise } \\
\hline Voltage & 23 & 23 & 23 & $\mathrm{nV} / \sqrt{ } \mathrm{Hz}$ \\
\hline Input-Bias Current & 140 & 140 & 140 & $\mathrm{nA}$ \\
\hline $\begin{array}{l}\text { Supply-Voltage } \\
\text { Range }\end{array}$ & $1-7.5$ & $1.8-7.5$ & $1-7.5$ & $\mathrm{~V}$ \\
\hline \multicolumn{5}{|l|}{ Total Supply } \\
\hline Current & 700 & 670 & 980 & $\mu \mathrm{A}$ \\
\hline CMRR & 100 & 100 & 100 & $\mathrm{~dB}$ \\
\hline Open-Loop Gain & 117 & 110 & 115 & $\mathrm{~dB}$ \\
\hline $\begin{array}{l}\text { Output-Voltage } \\
\text { Range }\end{array}$ & $\begin{array}{l}V_{C C}-0.1 / \\
V_{E E}+0.1\end{array}$ & $\begin{array}{c}V_{C C}-0.1 / \\
V_{E E}+0.1\end{array}$ & $\begin{array}{l}V_{C C}-0.1 / \\
V_{E E}+0.1\end{array}$ & V \\
\hline Output Current & \pm 15 & \pm 15 & \pm 15 & $\mathrm{~mA}$ \\
\hline Slew Rate & 1.1 & 1.1 & 0.9 & $V / \mu \mathrm{s}$ \\
\hline $\begin{array}{l}\text { Unity-Gain } \\
\text { Frequency }\end{array}$ & 3.4 & 3.5 & 2.7 & $\mathrm{MHz}$ \\
\hline $\begin{array}{l}\text { Unity-Gain Phase } \\
\text { Margin }\end{array}$ & 61 & 63 & 63 & degree \\
\hline
\end{tabular}

figure of merit $F_{M}$ than that the Darlington output and compared to the Widlar output, an improvement of almost a factor of 2 is achieved.

Table II lists typical overall specifications of the three op amps. The total supply current of the MPD op amp is $700 \mu \mathrm{A}$ and it is constant within $5 \%$ over the supply-voltage range from 1 to $7.5 \mathrm{~V}$. The average input-offset voltage is $\pm 0.6 \mathrm{mV}$, and the CMRR is $100 \mathrm{~dB}$ in the common-mode range where either the $n-p-n$ or the $p-n-p$ input pair is active and $62 \mathrm{~dB}$ in the turnover range of $Q_{550}$. If desired, this value can be enlarged at the cost of a proportional enlargement of the turnover range by placing a resistor in series with the emitter of $Q_{550}$. The maximum output current is $\pm 15 \mathrm{~mA}$. The slew rate of the op amp with MPD output is $1.1 \mathrm{~V} / \mu \mathrm{s}$.

\section{Conclusions and Discussion}

The operational amplifier that has been presented operates at supply voltages down to $1 \mathrm{~V}$, and it has rail-to-rail input and output ranges. A multipath-driven output stage is introduced with a feedforward path directly from the intermediate stage to the output transistor. It gives the op amp a bandwidth that is determined exclusively by the load capacitor and by the amount of current through the output transistors.

The op amp with multipath-driven output, as weil as the op amps with Darlington and Widlar outputs, have been implemented in a BiCMOS process with $3-\mathrm{GHz}$ n-p-n's and 1-GHz vertical p-n-p's. A figure of merit $F_{M}$ is established which is the ratio of bandwidth and power consumption of the output stages. The figures of merit for these three output stages are 18,11 , and $9.6 \mathrm{MHz} / \mathrm{mW}$, respectively. The difference between the three op amps is now mainly apparent at high-current levels. If lateral p-n-p's are used, the difference between the multipathdriven output stage and the Darlington and Widlar output stages is even more distinct. Even at low output-current levels, the output bump then occurs as a result of the internal poles of these latter output stages. The multipath-driven output stage directly addresses the output transistor and there is no output bump. Presently, a $1-\mathrm{V}$ $10-\mathrm{MHz}$ op amp, loaded by $10 \mathrm{pF}$ and using only $100-\mu \mathrm{A}$ quiescent current in the output stage with $100-\mathrm{MHz}$ lateral p-n-p's, is being developed. The figure of merit $F_{M}$ of this op amp is $120 \mathrm{MHz} / \mathrm{mW}$.

\section{ACKNOWLEDGMENT}

The authors wish to thank the people of Philips Nijmegen, The Netherlands, for processing the chips.

\section{REFERENCES}

[1] R. J. Widlar, "Low voltage techniques," IEEE J. Solid-State Circuits, vol. SC-13. pp. 838-846, Dec. 1978.

[2] J. E. Solomon, "The monolithic op amp: A tutorial study," IEEE J. Solid-State Circuits, vol. SC-9, no. 6, pp. 314-332, Dec. 1974.

[3] J. Fonderie, M. M. Maris, E. J. Schnitger, and J. H. Huijsing, "1-V operational amplifier with rail-to-rail input and output ranges," IEEE J. Solid-State Circuits, vol. 24, pp. 1551-1559, Dec. 1989.

[4] J. H. Huijsing and J. Fonderie, "Multi stage amplifier with capacitive nesting and multi-path-driven forward feeding for frequency compensation," U.S. Patent Appl. 654.855, Feb. 11, 1991.

[5] B. Y. Kamath, R. G. Meyer, and P. R. Gray, "Relationship between frequency response and settling time of operational amplifiers," IEEE J. Solid-State Circuits, vol. SC-9, pp. 347-352, Dec. 1974

[6] E. Seevinck, W. de Jager, and P. Buitendijk, "A low-distortion output stage with improved stability for monolithic power amplifiers," IEEE J. Solid-State Circuits, vol. 23, pp. 794-801, June 1988.

[7] J. H. Juijsing and D. Linebarger, "Low-voltage operational amplifier with rail-to-rail input and output ranges," IEEE J. Solid-State Circuits, vol, SC-20, pp. 1144-1150, Dec. 1985. 
[8] H. C. Nauta and E. H. Nordholt, "New class of high-performance PTAT current sources," Electron. Lett., vol. 21, pp. 384-386, 1985.

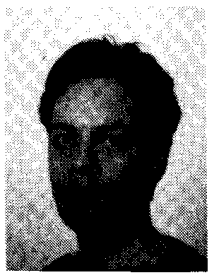

Jeroen Fonderie was born in Amsterdam, The Netherlands, on July 27, 1960 . He received the M.Sc. degree in electrical engineering from the Delft University of Technology, Delft, The Netherlands, in 1987 and the Ph.D. degree from the same university in 1991 .

Since 1987 he has been a Research Assistant at the Electronic Instrumentation Laboratory, Department of Electrical Engineering, Delft University of Technology, where he has been working on the subject of low-voltage opera-

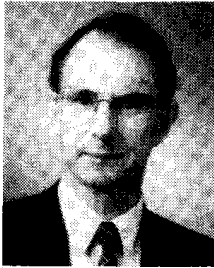

Johan H. Huijsing ( $\mathrm{SM}^{\prime} 81$ ) was born in Bandung, Indonesia, on May 21, 1938. He received the ingenieurs (M.Sc.) degree in electrical engineering from the Delft University of Technology, The Netherlands, in 1969 , and the Ph.D. degree from the same university in 1981 for work on operational amplifiers.

Since 1969 he has been a member of the Research and Teaching Staff of the electronic Instrumentation Laboratory, Department of Electrical Engineering, Delft University of Technology, as a full Professor. He is engaged in research on analog integrated circuits and integrated sensors for instrumentation systems.

tional amplifiers. 